\title{
On-Board Accurate Calibration of Dual-Channel Radiometers Using Internal and External References
}

\author{
Ignasi Corbella, Member, IEEE, Albin J. Gasiewski, Senior Member, IEEE, Marian Klein, Vladimir Leuski, \\ Anthony J. Francavilla, and Jeffrey R. Piepmeier, Member, IEEE
}

\begin{abstract}
This paper presents a method for combining internal noise injection and external reference standard looks to accurately calibrate an airborne dual-channel radiometer. The method allows real-time estimation of the correct values of the radiometer gains and offsets, even for nontemperature-stabilized radiometers and with minimum loss of measurement time spent in external load measurement. Crosstalk and leakage introduced by the noise injection circuitry is also taken into account, thus providing high gain and offset estimation accuracy. The method was implemented on a National Oceanic and Atmospheric Administration airborne instrument, the Polarimetric Scanning Radiometer, which was used to obtain an extensive set of radiometric measurements over oceanic convection during CAMEX3 in August-September 1998.
\end{abstract}

Index Terms-Calibration, radiometry.

\section{INTRODUCTION}

$\mathbf{M}$ ICROWAVE airborne or satellite radiometers are useful tools for obtaining geophysical parameters such as wind direction and velocity over the ocean, ice content, water vapor, sea surface salinity, soil moisture, vegetation cover, and others. By using pertinent models these parameters are estimated from the brightness temperature of the surface, which is measured by the radiometer. Since there is a linear dependence between the brightness temperature and the detected voltage, a radiometer is calibrated by measuring the thermal noise emitted from two sources having different and known emission temperatures. From these measurements the gain and the offset of the radiometer are estimated.

Calibration should be performed frequently enough so as to effectively track gain and offset variations. In many cases this

Manuscript received October 1999. This work was supported by the Spanish Ministerio de Educación y Cultura (Ref. PR1997-0047 0046214076), and by the Universitat Politècnica de Catalunya, Barcelona, Spain, while Prof. I. Corbella was on sabbatical leave at the U.S. National Oceanic and Atmospheric Administration.

I. Corbella was with the National Oceanic and Atmospheric Administration Environmental Technology Laboratory, Boulder, CO, 80303 USA, on leave from the Department of Signal Theory and Communications, Universitat Politecnica de Catalunya, Spain (e-mail: icorbella@ tsc.upc.es).

A. J. Gasiewski, M. Klein, V. Leuski, and A. J. Francavilla are with the National Oceanic and Atmospheric Administration/Environmental Technology Laboratory, Boulder, CO 80303 USA.

J. R. Piepmeier was with the School of Electrical Engineering and Computer Engineering, Georgia Institute of Technology Atlanta, GA 30332-0250 USA. He is now with the Microwave Instrument Technology Branch, NASA Goddard Space Flight Center, Greenbelt, MD 20771 USA.

Publisher Item Identifier 10.1109/TMTT.2002.800444. is impractical, especially if mechanical movement of the instrument is needed for measuring the reference targets. The classical solution is the use of a Dicke radiometer [1], which by quickly switching the receiver input from the antenna output to a reference load reduces the influence of gain variations in the fluctuations of the output voltage. This approach does not avoid the necessity of performing periodic calibrations with external sources, but it allows increasing the interval between them because the dependence of the system parameters is transferred from the gain and offset to other more stable parameters, depending of the specific scheme.

Since the Dicke switch has a duty cycle of $50 \%$, the effective integration time is cut in half its original value and the best radiometric resolution achievable with Dicke radiometers is degraded by a factor of two compared to a total power radiometer. Different duty cycles, in which the integration time assigned to measure the scene is increased, can eventually improve the radiometric sensitivity as long as the gain variations over the time interval measuring the scene are negligible. The calibration approach presented in this paper uses infrequent calibrations with external loads and periodic fast switching to noise diodes which are sequentially biased and unbiased.

This approach has been implemented in the Polarimetric Scanning Radiometer (PSR), described in the next section, and the results show that gain and offset variations are effectively cancelled out.

\section{THE POLARIMETRIC SCANNING RADIOMETER}

The PSR is a versatile airborne microwave imaging radiometer developed by the Georgia Institute of Technology (PSR/D) and the NOAA Environmental Technology Laboratory (PSR/A) for the purpose of obtaining polarimetric microwave imagery of the Earth's oceans, land, ice, clouds and precipitation. It consists of a set of microwave radiometers housed within a gimbal-mounted scanhead drum. This one is rotatable by a two-axis positioner so that the radiometers can view any angle within $70^{\circ}$ elevation of nadir at any azimuthal angle (a total of $1.32 \pi$ sr solid angle). The configuration supports conical, cross-track, along-track, fixed-angle stare, and spotlight scan modes. The entire assembly, including the scanhead with the radiometers and two passive calibration targets, is designed for integration into three NASA aircraft: the DC-8, Orion P-3B, and ER-2. 
TABLE I

PSR/A FREQUENCIES AND POLARIZATION CHANNELS

\begin{tabular}{cl}
\hline $\begin{array}{c}\text { Frequency } \\
\text { (GHz) }\end{array}$ & $\begin{array}{c}\text { Polarization } \\
\text { Channels }\end{array}$ \\
\hline $10.6-10.8$ & $\mathrm{~T}_{\mathrm{v}}, \mathrm{T}_{\mathrm{h}}, \mathrm{T}_{\mathrm{U}}, \mathrm{T}_{\mathrm{V}}$ \\
\hline $18.6-18.8$ & $\mathrm{~T}_{\mathrm{v}}, \mathrm{T}_{\mathrm{h}}, \mathrm{T}_{\mathrm{U}}, \mathrm{T}_{\mathrm{V}}$ \\
\hline $21.3-21.6$ & $\mathrm{~T}_{\mathrm{v}}, \mathrm{T}_{\mathrm{h}}$ \\
\hline $36.0-38.0$ & $\mathrm{~T}_{\mathrm{v}}, \mathrm{T}_{\mathrm{h}}, \mathrm{T}_{\mathrm{U}}$ \\
\hline $86.0-92.0$ & $\mathrm{~T}_{\mathrm{v}}, \mathrm{T}_{\mathrm{h}}, \mathrm{T}_{\mathrm{U}}$ \\
\hline
\end{tabular}

The PSR/A was assembled in 1998 at NOAA in preparation for the Third Convection and Moisture Experiment (CAMEX-3, August-September, 1998). During this campaign, the PSR/A was flown within a variety of meteorological conditions on more than 25 flights of $4-8 \mathrm{~h}$ each [2]. A list of channels and polarization specifications of PSR/A is shown in Table I.

\section{CALIBRATION USING EXTERNAL STANDARDS}

During normal operation the scanhead is periodically pointed to two different thermal targets made of absorbing material, one maintained at a constant temperature of $65{ }^{\circ} \mathrm{C}$ and the other at the local ambient temperature. Both are monitored to $0.1{ }^{\circ} \mathrm{C}$ precision and their values recorded. The operator selects the rate at which external calibration looks are performed, typically at about 1-2-min intervals, although for uninterrupted scanning during long flight lines, it can be even several tens of minutes. Several scans are typically completed between external calibration looks.

The hot and ambient loads are both measured within $5 \mathrm{~s}$, which is short compared to their several-minute thermal time constants. Each look lasts about 1/2 s and is an average over 30 samples.

At each calibration look, the gain and offset of the horizontally and vertically polarized channels are retrieved using the standard formulas

$$
\begin{aligned}
& g=\frac{v_{H}-v_{C}}{T_{H}-T_{C}} \\
& o=\frac{v_{C} T_{H}-v_{H} T_{C}}{T_{H}-T_{C}}
\end{aligned}
$$

where $v_{H}$ and $v_{C}$ are the detected output voltages when the hot and ambient targets are being measured, and $T_{H}$ and $T_{C}$ their emission temperatures. These ones are computed using their measured physical temperatures and their effective emissivities as determined by reference to cold sky looks.

Equation (1) allows estimation of the gain and offset of the radiometers only at the calibration times at which external target looks are performed. Between them, a simple linear interpolation is enough if the external calibration interval is short. Otherwise, internal noise-diode calibration between external looks is needed, extending the time interval between external calibration looks to up to $40 \mathrm{~min}$.

\section{Subinterval Calibration USING InTERnAL NOISE DIODES}

The internal calibration hardware used to supplement the standard hot- and cold-view calibration is depicted in Fig. 1.

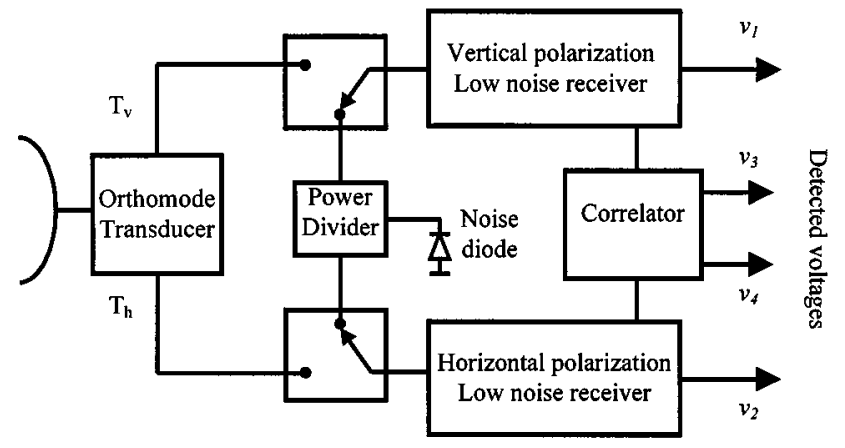

Fig. 1. Simplified bloc diagrams of the PSR/A radiometers showing the internal calibration hardware. The correlator is used to get the third and fourth Stokes parameters. These are not considered in the present work.

By pulsing the diode both on and off, it allows two-level noise power to be injected into the RF inputs of the receivers at 500-ms intervals. This time interval is considerably shorter than the one between views of the external calibration targets so as to allow estimation of the radiometer gains and offsets without breaking the sequence of raster scans and at time intervals comparable to the drift time of receivers.

Between diode on and off states there are about 35 Nyquist-rate samples. When the receivers inputs are switched to the antenna, each data sample is produced every $16-17 \mathrm{~ms}$ after averaging 32 voltage samples. When the input switch is connected to the diode (on or off), a sample is produced every $6 \mathrm{~ms}$ with an average value of 12, and each diode-on or diode-off look consists of three samples. Calibrating using the noise diodes relies on the accurate estimation of the "on-state" and "off-state" equivalent temperatures of the diodes. These ones drift with time as a function of their physical temperature and other conditions, so a dynamic on-board estimation of these parameters is required.

The detected output voltages of the radiometer (vertical or horizontal channel) when the diode is connected at the inputs are

$$
\begin{gathered}
v_{\mathrm{ON}}=g_{d} T_{\mathrm{ON}}+o_{d} \\
v_{\mathrm{OFF}}=g_{d} T_{\mathrm{OFF}}+o_{d}
\end{gathered}
$$

where $T_{\mathrm{ON}}$ and $T_{\mathrm{OFF}}$ are the equivalent noise temperatures at the switch output for each state. The gain and offset (indicated with subscript " $d$ ") are distinct from those computed in (1) because the effects of the antenna and circuits before the switch (i.e., transmission lines) have not been taken into account.

Relating one set to the other is straightforward: the overall receiver available power gain is simply given by

$$
g=g_{A} g_{d}
$$

where $g_{A}$ is the available power gain of the antenna path.

Using Friis formula (e.g., [3]), the equivalent noise temperature of the entire receiver referred to antenna terminals can be written as

$$
T_{R}=T_{A}+T_{R}^{d} / g_{A}
$$


where $T_{A}$ is the noise temperature of the antenna path and $T_{R}^{d}$ is the receiver noise temperature from the switch output. Since the radiometer offset is directly related to the receiver noise temperature by $o=g T_{R}$, (4) can be expressed directly in terms of it as follows:

$$
o=o_{d}+g_{d} g_{A} T_{A}
$$

where $o_{d}=g_{d} T_{R}^{d}$ is the offset when the diode is used.

Using (2)-(5) the receiver output voltages when the input is switched to the diode can be put explicitly as function of the total gain $(g)$ and offset $(o)$

$$
\begin{gathered}
v_{\mathrm{ON}}=g T_{\mathrm{ON}}^{\prime}+o \\
v_{\mathrm{OFF}}=g T_{\mathrm{OFF}}^{\prime}+o
\end{gathered}
$$

where $T_{\mathrm{ON}}^{\prime}$ and $T_{\mathrm{OFF}}^{\prime}$ are the effective diode ON and OFF noise temperatures referred to the antenna terminals, and are given by

$$
\begin{gathered}
T_{\mathrm{ON}}^{\prime} \triangleq T_{\mathrm{ON}} / g_{A}-T_{A} \\
T_{\mathrm{OFF}}^{\prime} \triangleq T_{\mathrm{OFF}} / g_{A}-T_{A} .
\end{gathered}
$$

The gain $g$ and the offset $o$ in (6) correspond to the overall receiver and are directly estimated from (1) at the time instances $t_{c}$ at which the radiometer is calibrated with external loads. Furthermore, at these times, the equivalent ON and OFF temperatures can be accurately estimated by solving (6)

$$
\begin{aligned}
\Delta T\left(t_{c}\right) & \triangleq T_{\mathrm{ON}}^{\prime}-T_{\mathrm{OFF}}^{\prime}=\frac{v_{\mathrm{ON}}\left(t_{c}\right)-v_{\mathrm{OFF}}\left(t_{c}\right)}{g\left(t_{c}\right)} \\
T_{\mathrm{OFF}}^{\prime}\left(t_{c}\right) & =\frac{v_{\mathrm{OFF}}\left(t_{c}\right)-o\left(t_{c}\right)}{g\left(t_{c}\right)} .
\end{aligned}
$$

Assuming now that these equivalent diode noise temperatures are slowly varying functions of time, their values between calibrations can be estimated by linear interpolation. From them and using (6), the gain and offset can be computed at the high time rate defined by the diode switching.

Fig. 2 shows the gain and offset of the $18.7-\mathrm{GHz}$ receiver computed from external calibration (circles) and also from the noise diode voltages (continuous line) using the above method. It is clear that the procedure can track the rapid gain and offset variations for which simple linear interpolation between gain and offset at sample times would fail.

\section{CRosstalk}

Due to nonideal hardware, when the radiometers inputs are switched to the noise diode, there may exist leakage from the signal coming from the antenna and crosstalk between polarizations. This was the case of the $89-\mathrm{GHz}$ PSR/A radiometer during the CAMEX-3 campaign. It used magnetically switched circulators as switches, so when the diode signal was imputed to the receivers (position illustrated in Fig. 1) the antenna outputs were also connected to the power divider, which was made of a simple waveguide " $T$." The result was a fairly high cross talk between the horizontal and vertical channels during internal calibration.

Although new versions of the PSR include isolators to avoid this effect, the large amount of data collected with the hardware made it necessary to devise a method to estimate the

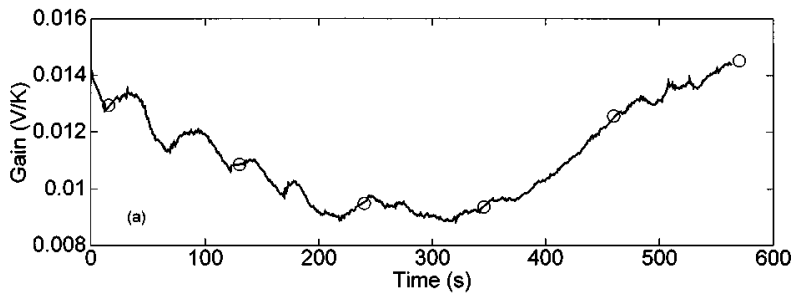

(a)

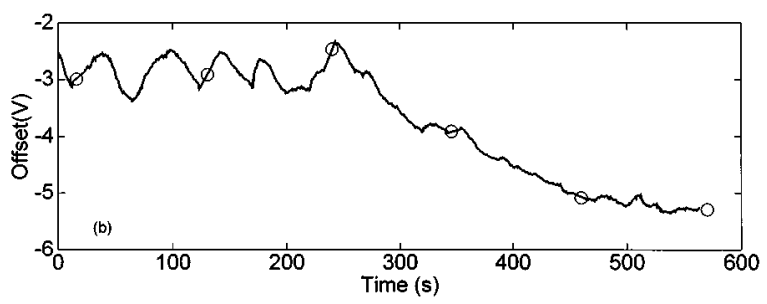

(b)

Fig. 2. (a) Computed gain and (b) offset from noise diodes internal calibration $(-)$ and from hot/cold external looks (o).

crosstalk terms and compensate them, making it possible to use the subinterval calibration described in the previous section. Both crosstalk and leakage can be included in (6), which now becomes (split for vertical and horizontal polarizations)

$$
\begin{gathered}
v_{v \mathrm{ON}}=g_{v} T_{v \mathrm{ON}}^{\prime}+o_{v}+g_{v} \alpha_{v v} T_{v}+g_{v} \alpha_{v h} T_{h} \\
v_{v \mathrm{OFF}}=g_{v} T_{v \mathrm{OFF}}^{\prime}+o_{v}+g_{v} \alpha_{v v} T_{v}+g_{v} \alpha_{v h} T_{h} \\
v_{h \mathrm{ON}}=g_{h} T_{h \mathrm{ON}}^{\prime}+o_{h}+g_{h} \alpha_{h v} T_{v}+g_{h} \alpha_{h h} T_{h} \\
v_{h \mathrm{OFF}}=g_{h} T_{h \mathrm{OFF}}^{\prime}+o_{h}+g_{h} \alpha_{h v} T_{v}+g_{h} \alpha_{h h} T_{h}
\end{gathered}
$$

where additional subscripts $v$ and $h$ have been used to indicate the polarization, and $T_{v}$ and $T_{h}$ denote the vertical and horizontal scene brightness temperatures. Crosstalk is accounted for by the coefficients $\alpha_{v h}$ and $\alpha_{h v}$ and leakage by $\alpha_{v v}$ and $\alpha_{h h}$.

From the above it is apparent that the differences between the $\mathrm{ON}$ and OFF voltages $\left(v_{v \mathrm{ON}}-v_{v \mathrm{OFF}}\right.$ and $\left.v_{h \mathrm{ON}}-v_{h \mathrm{OFF}}\right)$ are unaffected by cross talk or leakage, thus (8) remains valid, and also the estimation of the gains $g_{v}$ and $g_{h}$ being both only function of voltage differences.

The offset estimation however is affected since (9) is no longer valid. Now $T_{\mathrm{OFF}}^{\prime}$ must be estimated by solution of the complete equation (10) at the calibration times $t_{c}$ as follows:

$$
\begin{aligned}
v_{v \mathrm{OFF}}\left(t_{c}\right)= & g_{v}\left(t_{c}\right) T_{v \mathrm{OFF}}^{\prime}\left(t_{c}\right)+o_{v}\left(t_{c}\right) \\
& +\alpha_{v v}\left[v_{v}\left(t_{c}\right)-o_{v}\left(t_{c}\right)\right] \\
& +\alpha_{v h} \frac{g_{v}\left(t_{c}\right)}{g_{h}\left(t_{c}\right)}\left[v_{h}\left(t_{c}\right)-o_{h}\left(t_{c}\right)\right] \\
v_{h \mathrm{OFF}}\left(t_{c}\right)= & g_{h}\left(t_{c}\right) T_{h \mathrm{OFF}}^{\prime}\left(t_{c}\right)+o_{h}\left(t_{c}\right) \\
& +\alpha_{h v} \frac{g_{h}\left(t_{c}\right)}{g_{v}\left(t_{c}\right)}\left[v_{v}\left(t_{c}\right)-o_{v}\left(t_{c}\right)\right] \\
& +\alpha_{h h}\left[v_{h}\left(t_{c}\right)-o_{h}\left(t_{c}\right)\right]
\end{aligned}
$$

where the voltages $v_{v}$ and $v_{h}$ correspond to the antenna viewing the scene at the times adjacent to the diodes measurement. The above equations can be easily solved for $T_{v \mathrm{OFF}}^{\prime}\left(t_{c}\right)$ and $T_{h \mathrm{OFF}}^{\prime}\left(t_{c}\right)$ provided the crosstalk and leakage coefficients are known. 


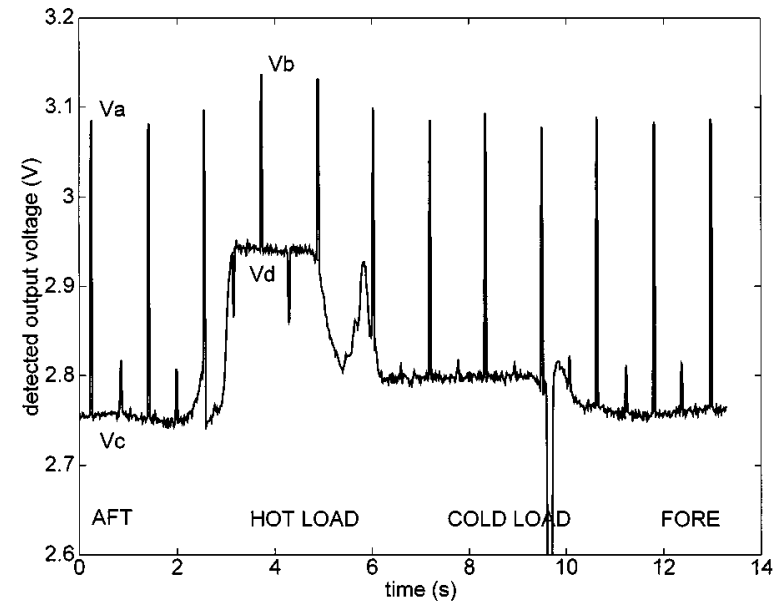

Fig. 3. Raw output voltage of the $89-\mathrm{GHz}$ vertical receiver during one external calibration look. The scanhead position at different times is specified in the bottom. The spikes in the voltage show the noise diode measurements. For crosstalk free receivers $V_{a}$ and $V_{b}$ should be the same.

The offset of both receivers $o_{v}$ and $o_{h}$ can now be estimated solving (10) which can be cast as a linear system

$\left[\begin{array}{cc}1-\alpha_{v v} & -\alpha_{v h} g_{v} / g_{h} \\ -\alpha_{h v} g_{h} / g_{v} & 1-\alpha_{h h}\end{array}\right]\left[\begin{array}{c}v_{v}-o_{v} \\ v_{h}-o_{h}\end{array}\right]=\left[\begin{array}{c}v_{v}-o_{v}^{\prime} \\ v_{h}-o_{h}^{\prime}\end{array}\right]$

being

$$
\begin{aligned}
& o_{v}^{\prime}=v_{v \mathrm{OFF}}-g_{v} T_{v \mathrm{OFF}}^{\prime} \\
& o_{h}^{\prime}=v_{h \mathrm{OFF}}-g_{h} T_{h \mathrm{OFF}}^{\prime} .
\end{aligned}
$$

\section{A. Crosstalk and Leakage Terms Estimation}

The crosstalk and leakage terms can be estimated from the raw output voltages by identifying data for which an abrupt change in input brightness temperature occurred, for example in transitions between the hot load, cold load and scene. During these changes, even when the inputs are switched to the diodes, the radiometer output voltages vary due to crosstalk.

Fig. 3 shows the raw output voltage for the $89-\mathrm{GHz}$ vertical receiver during one external calibration look. The spikes are the diode $\mathrm{ON}$ and OFF measurements, while the remainder of the points corresponds to the switch being connected to the antenna. The voltages labeled $V_{a}$ and $V_{b}$ both correspond to the diode ON connected at the input, but for the first one the antenna is looking to the scene and for the second one it is measuring the external hot target. In an ideal system $V_{a}$ and $V_{b}$ should be the same, but a sensible difference between both is observed.

Using (10), this voltage difference may be computed in terms of the crosstalk and leakage coefficients

$$
\Delta v_{v o n}=V_{b}-V_{a}=g_{v} \alpha_{v v} \Delta T_{v}+g_{v} \alpha_{v h} \Delta T_{h}
$$

where $\Delta T_{v}$ and $\Delta T_{h}$ are the vertical and horizontal brightness temperature difference between the scene and the hot load. On the other hand, when the input switch selects the antenna (for example, points $V_{c}$ and $V_{d}$ ) the voltage difference is simply given by

$$
\Delta v_{v}=V_{d}-V_{c}=g_{v} \Delta T_{v}
$$

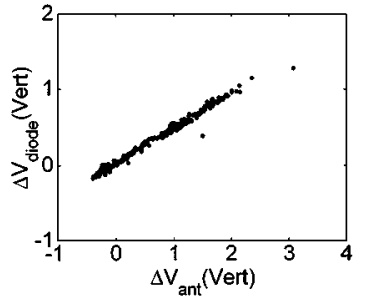

(a)

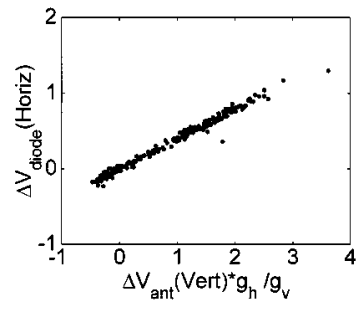

(c)

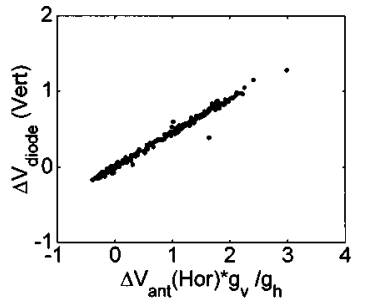

(b)

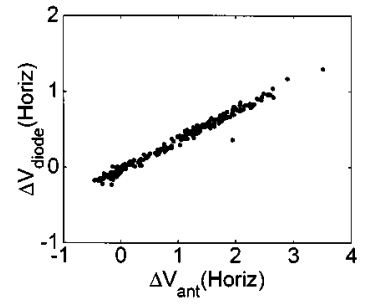

(d)
Fig. 4. Measured voltage increments $\left(V_{a}-V_{b}\right.$ vs. $\left.V_{d}-V_{c}\right)$ of the $89-\mathrm{GHz}$ receiver for an entire PSR/A flight. The expected linear dependence is apparent.

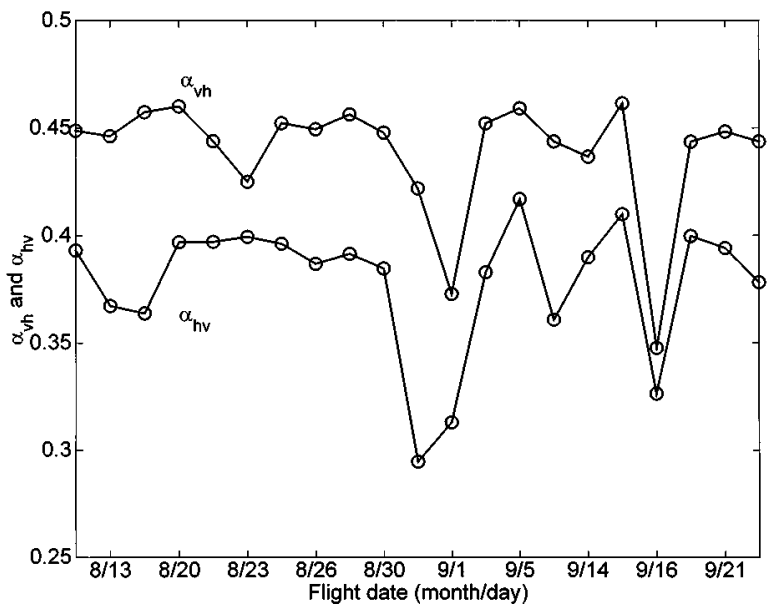

Fig. 5. Crosstalk coefficients of the $89-\mathrm{GHz}$ receiver retrieved for all CAMEX3 flights. High consistency among different dates is observed.

Now, by inserting (15) into (14) the first of the following equations is obtained. The second one is for the horizontal receiver

$$
\begin{aligned}
& \Delta v_{v \mathrm{ON}}=\alpha_{v v} \Delta v_{v}+\alpha_{v h} g_{v} / g_{h} \Delta v_{h} \\
& \Delta v_{h \mathrm{ON}}=\alpha_{h v} g_{h} / g_{v} \Delta v_{v}+\alpha_{h h} \Delta v_{h} .
\end{aligned}
$$

The ratio $g_{v} / g_{h}$ appearing in the above equations is computed from the external hot and cold target measurements

$$
\frac{g_{v}}{g_{h}}=\frac{v_{v \mathrm{HOT}}-v_{v \mathrm{COLD}}}{v_{h \mathrm{HOT}}-v_{h \mathrm{COLD}}} .
$$

Fig. 4 shows the measured voltage increments, as defined above, for the entire PSR/A flight on September 21, 1998. The linear dependence between the diode-switched voltages and the antenna-switched voltages predicted in (16) is clearly seen. Now, from a large number of such $\Delta v$ measurements the crosstalk terms $\alpha_{v h}$ and $\alpha_{h v}$ and the leakage terms $\alpha_{v v}$ and $\alpha_{h h}$ were statistically estimated by minimizing the difference between the measured voltage increments and the ones predicted by (16) using linear minimum mean square error estimation [4]. From the data in Fig. 4, the values $\alpha_{v v}=0.0344 \alpha_{v h}=0.42$ 
$\alpha_{h v}=0.4$ and $\alpha_{h h}=-0.0006$ were obtained, which are consistent with theoretical calculations obtained using manufacturer stated specifications for component parameters. Note that the cross talk terms are an order of magnitude higher than the leakage terms, as it was already expected.

This procedure was carried out for over 100 hours of PSR/A flights during 21 sorties and shows very consistent behavior for the entire CAMEX data set, as shown in Fig. 5.

\section{CONCLUSION}

A new technique for radiometer calibration using combined external and internal references has been presented. The technique was implemented for processing data from the NOAA PSR/A obtained during a number of flights over the ocean during the CAMEX-3 campaign in 1998. The method consists of periodically switching the receivers' input to an $\mathrm{ON}-\mathrm{OFF}$ switched noise diode, combined with infrequent views at external reference targets. An algorithm has been developed to compute the gain and offset at closely spaced time intervals using the noise diodes, but referring the diodes' noise temperatures to precise measurements of the external targets. Crosstalk and leakage introduced by the diode circuitry in one of the receivers was taken into account to improve the calibration accuracy. The results show that gain and offset variations can be accurately tracked with the diodes used as fast transfer standards. The method is well suited for use in a variety of radiometric applications.

\section{REFERENCES}

[1] F. T. Ulaby, R. K. Moore, and A. K. Fung, Microwave Remote Sensing, Active and Passive. Norwood, MA: Artech House, 1981, vol. I, sec. 6-9.

[2] D. J. Serke, A. J. Gasiewski, M. Klein, V. Leusky, A. Frankavilla, J. R. Piepmeier, and I. Corbella, "Processing and initial comparison of PSR data from CAMEX-3 to SSM/I and TMI data," in Proc. IEEE Int. Geosci. Remote Sensing Symp., vol. 1, Hamburg, Germany, July 1999, pp. 243-246.

[3] A. B. Carlson, Communication Systems, 2nd ed. New York: McGrawHill, 1975.

[4] A. Papoulis, Probability, Random Variables, and Stochastic Processes. New York: McGraw-Hill, 1965.

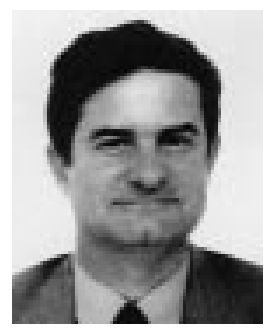

Ignasi Corbella (M'99) received the Enginyer and Doctor Enginyer degrees in Telecommunication Engineering from the Universitat Politècnica de Catalunya (UPC), Barcelona, Spain, in 1977 and 1983, respectively.

In 1976, he joined the School of Telecommunication Engineering, Barcelona, Spain, as a Research Assistant in the Microwave Laboratory, were he worked on passive microwave integrated circuit design and characterization. During 1979, he was with Thomson-CSF, Paris, France, where he was involved with microwave oscillators design. In 1982, he became an Assistant Professor with UPC, an Associate Professor in 1986, and a Full Professor in 1993. He currently teaching microwaves at an undergraduate level and has designed and taught graduate courses on nonlinear microwave circuits. From 1993 to 1997, he was Vice-Dean of the School of Telecommunications Engineering. During the 1998/1999 school year, he was with the U.S. National Oceanic and Atmospheric Administration (NOAA)/Environmental Technology Laboratory, Boulder, $\mathrm{CO}$, where he was a Guest Researcher involved with the development of methods for radiometer calibration and data analysis. He is currently Director of the Department of Signal Theory and Communications, UPC. His research with the Department of Signal Theory and Communications includes microwave airborne and satellite radiometry and microwave system design.

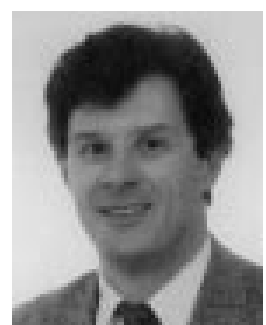

Albin J. Gasiewski (S'81-M'88-SM'95) received the M.S. and B.S. degrees in electrical engineering and the B.S. degree in mathematics from Case Western Reserve University, Cleveland, $\mathrm{OH}$, in 1983, and the Ph.D. degree in electrical engineering and computer science from the Massachusetts Institute of Technology, Cambridge, in 1989.

From 1989 to 1997 , he was a faculty member within the School of Electrical and Computer Engineering, Georgia Institute of Technology, Atlanta. As an Associate Professor with the Georgia Institute of Technology, he developed and taught courses on electromagnetics, remote sensing, instrumentation, and wave propagation theory. He is currently with the Environmental Technology Laboratory (ETL), U.S. National Oceanic and Atmospheric Administration (NOAA) Boulder, CO, where he is Acting Chief of the ETL Microwave Systems Development Division. His technical interests include passive and active remote sensing, radiative transfer theory, electromagnetics, antennas and microwave circuits, electronic instrumentation, meteorology, and oceanography.

Dr. Gasiewski is a member of Tau Beta Pi, Sigma Xi, the American Meteorological Society, the American Geophysical Union, and the International Union of Radio Scientists (URSI). He is a member of the Administrative Committee (AdCom) IEEE Geoscience and Remote Sensing Society. He was the general chair of the 2nd Combined Optical-Microwave Earth and Atmosphere Sensing Symposium (CO-MEAS 1995). He organized the Technical Program for the 20th International Geoscience and Remote Sensing Symposium (IGARSS 2000). He is the the general co-chair of IGARSS 2006, Denver, CO. He is currently secretary of USNC/URSI Commission F. He served on the U.S. National Research Council Committee on Radio Frequencies (CORF) from 1989 to 1995.

Marian Klein, photograph and biography not available at time of publication.

Vladimir Leuski, photograph and biography not available at time of publication.

Anthony J. Francavilla, photograph and biography not available at time of publication.

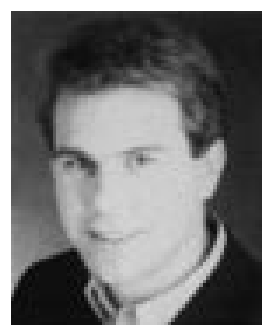

Jeffrey R. Piepmeier (S'90-M'99) received the B.S. degree in engineering from LeTourneau University, Longview, TX, in 1993, and the M.S. and Ph.D. degrees in electrical engineering from the Georgia Institute of Technology, Atlanta, in 1994 and 1999, respectively.

From 1993 to 1994, he was a Schakleford Fellow with the Georgia Institute of Technology Research Institute. In 1999, he joined the Microwave Instrument Technology Branch, NASA Goddard Space Flight Center, Greenbelt, MD. His technical interests include passive remote sensing, technology development for microwave radiometry and polarimetry, and high-speed digital and analog circuits.

Dr. Piepmeier is a member of URSI (Commission F) and the American Geophysical Union. He was conference chair of the 2000 Microwave Radiometer Calibration Workshop (MicroCal2000). He was the third place winner of the 1998 IGARSS student prize paper competition. He was also the recipient of an Excellence in Federal Career Gold Award (Rookie of the Year) in 2000. 